\title{
Customization and Automation of Data Acquisition and Evaluation using DigitalMicrograph Script
}

\author{
Bernhard Schaffer
}

Gatan R\&D/Gatan Inc., Pleasanton, USA

The Gatan Microscopy Suite (GMS) contains a free [1], powerful scripting language generally referred to as 'DigitalMicrograph Script' or simply 'DM Script' [2]. It is run within the main DigitalMicrograph application and gives easy access to most of the applications functionality. Its syntax is simplified and geared toward easy image processing, but also has features associated with mature programming languages such as object oriented programming.

Being an integral part of the application, DM Script is the ideal tool to tweak or extend DigitalMicrograph's behavior to personal needs, often using only a few lines of code. Typical uses range from automatic arrangements of images windows within the application, to batch-processing of data on the hard drive, to new data inspection tools for multidimensional spectrum-image data, for example. While not necessarily of scientific interest in themselves, such simple scripts can be enormous timesavers and thereby allow analysis of large amounts of data often needed for statistical verification. Furthermore, scripting offers data extraction, correction and evaluation possibilities beyond the provided application tools [3-5]. As analysis routines are written and used directly within DigitalMicrograph often the application with which the data is acquired - immediate results can also provide essential feedback and assistance during data acquisition sessions. DM Script is also a powerful tool to perform customized experiments. Access to Gatan's hardware (various cameras, DigiScan beam scanning unit, energy filter or spectrometer) and parts of the host microscope opens the route to in-house technique development as evident from a range of publications [6-11].

In this presentation we will demonstrate the capabilities of DM Script using a range of examples from simple application tools to complex data acquisition and evaluation scripts giving access to improved data quality.

\section{References:}

[1] Offline copies of DigitalMicrograph ${ }^{\circledR}$ are freely available for both 32-bit and 64-bit systems.

Please see http://www.gatan.com/products/software/ for details.

[2] DRG Mitchell and B Schaffer, Ultramicroscopy 103 (2005), p.319

[3] DRG Mitchell, Microscopy Research and Technique 71/8 (2008), p.588

[4] CH Wu et al., Ultramicroscopy 112/1 (2012), p.10

[5] N Braidy et al., Ultramicroscopy 118 (2012), p.67

[6] B Schaffer et al., Ultramicroscopy 106/8 (2006), p.1129

[7] M Bosman and VJ Keast, Ultramicroscopy 108/9 (2008), p.837

[8] T Heil and H Kohl, Ultramicroscopy 110/7 (2010), p.745

[9] K Sader et al., Ultramicroscopy 110/8 (2010), p.998

[10] P Potapov and HJ Engelmann, Ultramicroscopy 111/12 (2011), p.1681

[11] V Kumara, Microscopy and Microanalysis 17/6 (2011), p.859 
a)

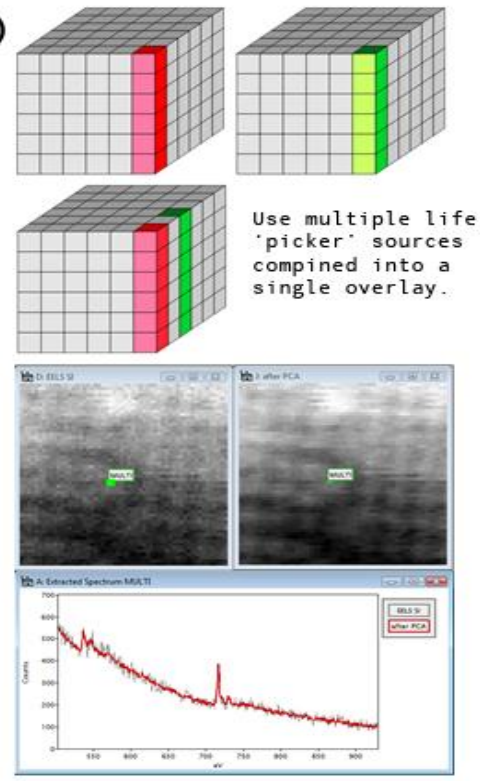

b)

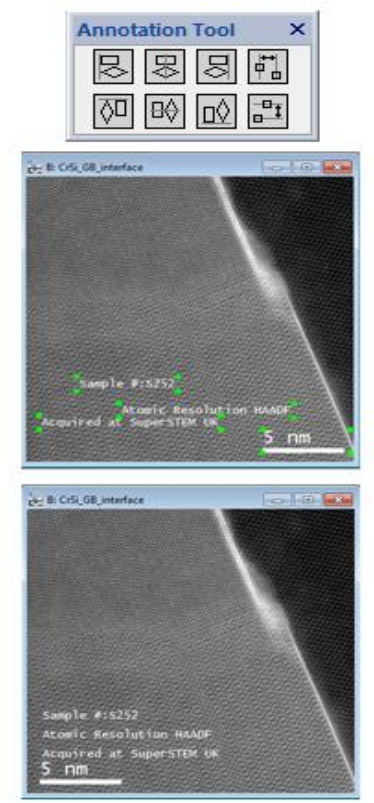

c)

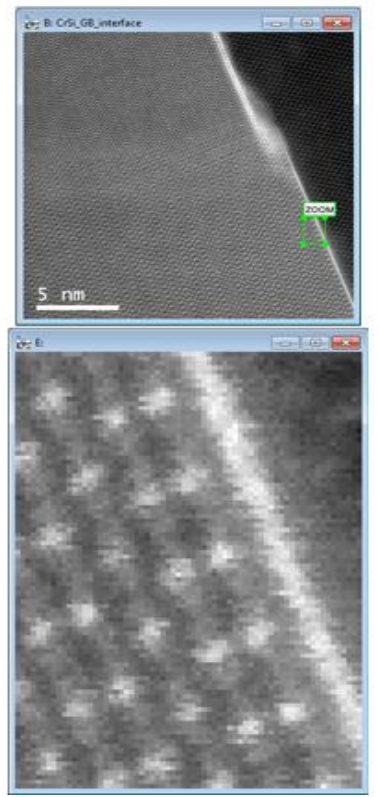

Figure 1. Examples of scripts adding new tool functionality to DigitalMicrograph: a) SI inspection tool to conveniently compare multiple spectra from different datasets; b) Annotation alignment tool; c) Interactive magnifying glass tool.

a)

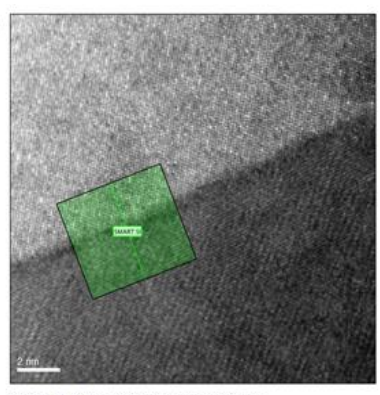

STEM HAADF image, random GB

b)
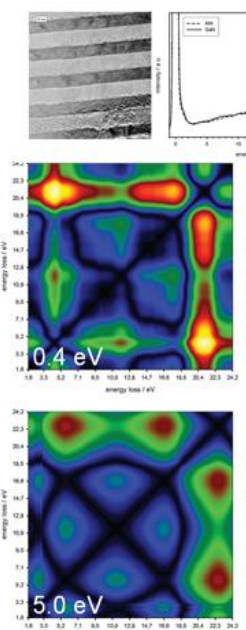

Plasmon ratio contrast maps
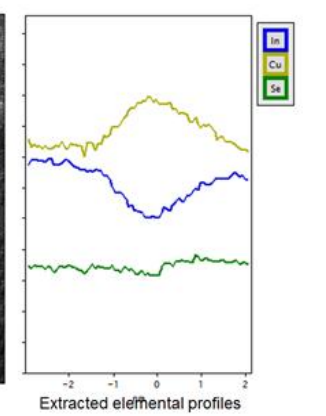
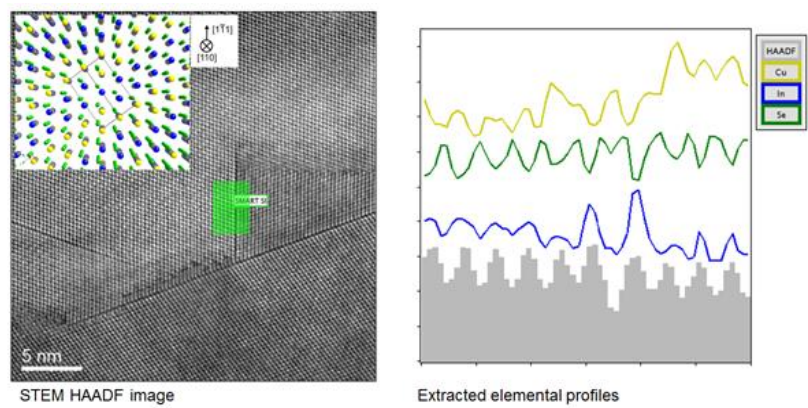

Extracted elemental profiles

c)
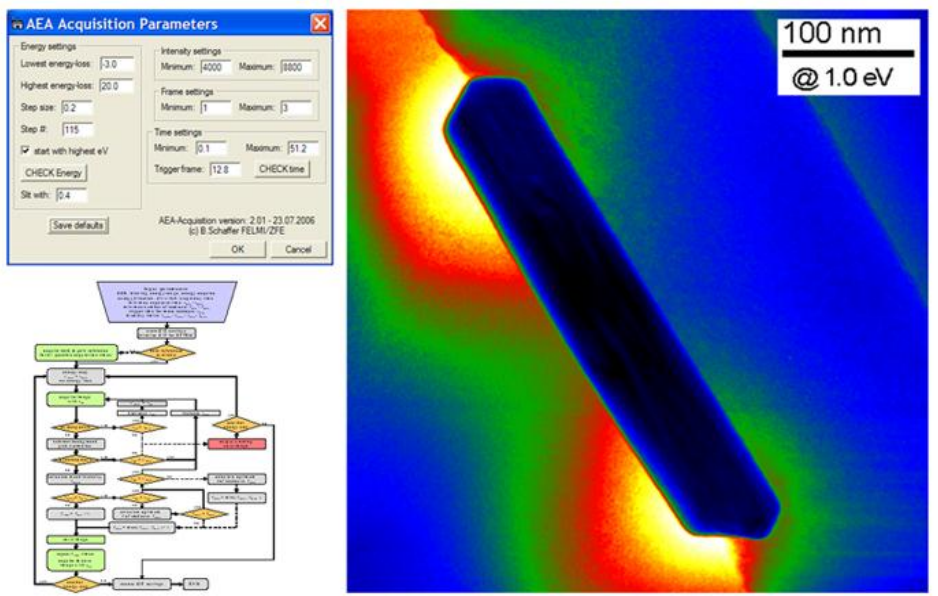

Figure 2. Examples of technique development by scripting: a) SMART acquisition: Atomic resolution STEM SI with rapid 1D averaging; b) Automated EFTEM jump-ratio contrast optimization; c) High energy-resolution EFTEM SI acquisition 